\title{
FUERZA LABORAL DE LA MUJER EN CHILE: CIFRAS Y CARACTERÍSTICAS
}

Jasna Stiepovich Bertoni*

BERTONI, J.S. Fuerza laboral de la mujer en Chile: cifras y caracteristicas. Rev. latino-am.enfermagem, Ribeirão Preto, v. 6, n. 5, p. 53-58, dezembro 1998.

El presente artículo muestra algunas características sociodemográficas de la fuerza laboral femenina en Chile y las dificultades que las mujeres deben enfrentar para acceder y mantenerse en su fuerza laboral, dada su condición de género.

TÉRMINOS CLAVES: mujer, fuerza laboral, Chile

\section{INTRODUCCIÓN}

Históricamente la evolución de la división del trabajo por sexo llevó a la diversificación de tareas entre hombres y mujeres que se expresa claramente en la asignación de roles específicos para cada uno de ellos. Así las tareas vinculadas a la producción han sido de responsabilidad de los hombres en el mundo público, para dar cumplimiento a su misión de proveedor al seno de la familia, teniendo una limitada participación en el mundo privado.

La mujer en Chile, constituye el $50.6 \%$ de la población del país (6.795.147) según último censo poblacional de $1992^{3}$. Aún en nuestra sociedad a la mujer se la vincula al espacio privado, siendo la función prioritaria atribuida a la mujer, el cuidado de los hijos y los quehaceres domésticos; tareas de la reproducción social. Durante el año $1994^{4}$, el 59\% de las mujeres entre 20 y 64 años, estaban fuera de la fuerza laboral; distribuyéndose en $54.1 \%$ en quehaceres del hogar, $2.9 \%$ como estudiantes, $0.6 \%$ como jubiladas, igual porcentaje como incapacitadas y un $0.8 \%$ en otras funciones. Por tanto, los quehaceres domésticos constituyen la actividad principal de la mayoría de las mujeres entre 20 y más años.

Las mujeres que conforman la fuerza laboral del país, ha ido en aumento con el transcurso de los años, siendo la tasa de participación en los años 1970 de 24.1, en 1985 de 29.8 , en 1990 de 31.8 , ascendiendo a un $41 \%$ en 1994 en edades entre 20 y 64 años ${ }^{4,5}$.

Las estadísticas muestran un incremento sostenido de la tasa ocupacional femenina en los últimos años, constatándose que un importante sector poblacional femenino, se vincula a producción nacional intentando compatibilizar actividades del ámbito privado con nuevas actividades del mundo público. Surge así la necesidad de recabar información respecto de la participación femenina en la fuerza de trabajo respecto de sus características sociodemográficas, categorías ocupacionales y las dificultades que se perciben al integrarse a la vida laboral del país.

\section{ANTECEDENTES SOCIODEMOGRÁFICOS}

En Chile, a partir de la década del 50, con una población urbana de un $60 \%$, se produce una importante tendencia de movilización de zonas rurales a urbanas, alcanzando la población urbana a un $83.46 \%$ en 1992 , segun censo poblacional ${ }^{3}$.

En este proceso migratorio, las mujeres fueron mayoritarias, dando como resultado que la población femenina rural sea menor a la masculina, $7.6 \%$ y $8.9 \%$ respectivamente. La mujer inicia el movimiento migratorio más temprano que los varones generalmente entre $15 \mathrm{y}$ 19 años y por plazos largos terminando muchas veces con el desarraigo del campo. Esta situación podría estar influenciando que en el caso específico de la fuerza de trabajo femenina, casi la mitad de ella se concentra en la capital del país, $49.34 \%$; aspecto que podría relacionarse también con las tasas de desocupación en áreas urbanas donde la femenina es de 7.0 siendo 9 puntos superior a la masculina, entre octubre a diciembre de $1994^{4}$.

El $41 \%$ de las mujeres y el $90 \%$ de los hombres están en el mercado de trabajo en edades entre 20 y 64 años ${ }^{4}$. Del total de mujeres el $38.4 \%$ y el $85.1 \%$ de los

* Profesor Titular. Departamento de Enfermería. Universidad de Concepción; Magister Enfermería Médico-Quirúrgica, Doctoranda Programa Doctorado Interunidades USP - Brasil 
hombres se encuentran en la categoría de ocupados, desarrollando un trabajo remunerado, durante el año 1994.

Entre los desocupados, buscan empleo por primera vez el $0.4 \%$ de la población femenina e igual porcentaje de hombres. En la categoría de cesantes se encuentra el $2.2 \%$ de mujeres y $4.5 \%$ de hombres.

Se hace necesario destacar que existe un porcentaje de población femenina aún no cuantificada, que está adscrita al mundo de la producción informal, producción subterránea de carácter inestable, desprotegida de toda seguridad social, donde se remunera parte del trabajo sin considerar local, insumos,seguridad entre otros; como también es posible de observar ambientes de trabajo de riesgo para la salud de las trabajadoras.

Es evidente la existencia de una pirámide de subcontratación en la mano de obra femenina. Investigadores en $1994^{7}$, realizaron un estudio sobre empleo en mujeres inactivas. Un importante grupo de mujeres 873.514 , que se declararon «inactivas» en encuesta del Instituto Nacional de Estadística ${ }^{1}$ sobre empleo en julio/septiembre de 1993, habían trabajado en algún período del año anterior e incluso en el momento mismo de la entrevista estaban realizando algún trabajo que no lo declararon en la encuesta por considerarlo poco significativo o por no diferenciarlo del trabajo doméstico.

El subempleo se caracteriza por ser en su mayoría un trabajo industrial no calificado, con uso intensivo de mano de obra y con escasas herramientas. Se identifican actividades tales como: armado de cajas de cartón, limpieza y selección de productos agrícolas como porotos y lentejas, envasado de condimentos de cocina, terminación y envasado de juguetes de plásticos, confección de prendas de vestir, entre otros ${ }^{6}$.

En educación, la tasa de alfabetismo a nivel nacional es de $94.5 \%$, la tasa de escolaridad tiene una cobertura en la educación básica de un $95 \%$ y en la educación media del 80\%. En la educación universitaria se estima que la cobertura corresponde a un $30 \%$ de la población que egresa de la enseñanza media. Al analizar la condición de alfabetismo por sexo en 1994, se puede apreciar una escasa diferencia, siendo para los hombres de un $94.78 \%$ y para las mujeres de un $94.43 \%{ }^{4}$.

La tasa de participación de la mujer en la fuerza productiva está estrechamente ligada a los años de estudio, así las mujeres con 8 o menos, participan en un $26.8 \%$, las que tienen entre 9 a 12 años de estudio lo hacen en un $36 \%$ y las que han cursado 13 o más años se incorporan en un $57.3 \%$. Las analfabetas trabajan en un $15 \%$.

Por tanto la tasa de participación de las mujeres en la fuerza de trabajo aumenta fuertemente con su nivel educacional, desde un $17 \%$ para las que tienen 3 y menos años de estudio, hasta $69 \%$ para las que aprobaron 16 y más años ${ }^{4}$. Es importante destacar que las mujeres en la fuerza laboral tienen más años de estudio que los hombres activos.

Debido a las altas tasas de participación laboral masculina, no hay grandes diferencias en el nivel educacional de activos e inactivos. Entre mujeres, las activas tienen un nivel de educación superior que las inactivas ${ }^{4}$.

En los últimos 25 años hubo un cambio en el comportamiento reproductivo de la población. El valor actual de la tasa global de fecundidad es inferior en más de 2 hijos por mujer. Chile se sitúa en la actualidad junto a Uruguay y Cuba entre los países de más baja fecundidad en Latinoamérica, así como también dentro de aquellos de más rápido descenso en todos los estratos sociales, sin embargo la fecundidad más alta ha estado asociada con aquellas ocupaciones relacionadas con las actividades primarias, en tanto, las más bajas se asocian con ocupaciones profesionales, empleados de oficina y obreros en industrias urbanas ${ }^{4}$.

Los hijos son una variable importante que incide en la participación femenina ocupacional, observándose tasas de participación de mujeres en edad reproductiva; entre el grupo 25-29 años, la tasa de participación es de 27 para las que tienen hijos y de 68 para las que no tienen entre 30 y 34 años la tasa de participación es de 30 y 60 respectivamente; entre 35 y 39 años las tasas para las mujeres con hijos es de 34 y para las sin hijos de 65; entre 40 y 44 años las tasas son de 35 y 61 con las con y sin hijos respectivamente. En todas las edades, las mujeres sin hijos tienen tasas de participación laboral que duplica la de mujeres con hijos ${ }^{3}$.

Las ramas de actividad económica en que las mujeres se desempeñan preferentemente ${ }^{8}$, muestran una participación mayoritaria en el sector servicios comunales, sociales y personales, con un $46.44 \%$. En segundo lugar participan en el comercio con un $26.46 \%$, como vendedoras, atención de hoteles, restaurantes y fuentes de soda. La industria manofacturera concentra el 14.18\% de la mano de obra femenina. En la agricultura alcanza un $6.03 \%$ de la población económicamente activa nacional, en tanto que en el sector financiero este es de un 5.72\%. Cinco son los rubros de actividad femenina predominante, los que en su conjunto agrupan al $96.83 \%$ de las mujeres en el mercado laboral. Siendo el sector terciario de la economía el que las agrupa en forma mayoritaria, particularmente en servicios y comercio en un $70.9 \%$.

Respecto de las ocupaciones que las mujeres desempeñan en cada rama de actividad, existen diferencias importantes si se las compara con la de los hombres. En el sector servicios, más de la mitad de las mujeres se desempeñan como "personal de servicio», (trabajadoras de casas particulares, personal de aseo). En segundo lugar, las mujeres del sector servicios se desempeñan como profesionales, siendo en este sector donde se registra el más alto porcentaje de profesionales 
y técnicas. Luego están las que trabajan como empleadas de oficina, cuyo porcentaje es también significativo.

En el comercio, la categoría ocupacional más relevante es la de vendedores, tanto para mujeres como para hombres. Posteriormente le siguen las que se desempeñan como empleadas de oficina y en tareas de servicios menores, como labores de aseo, cocina, atención de público en restaurantes y otros. En este sector se da también, un pequeño porcentaje de mujeres que se desempeñan como empresarias, $6 \%$ aproximadamente. Es necesario destacar que el porcentaje de mujeres que ocupan cargos directivos en esta rama es mayor que en las otras.

En la industria manofacturera, la mano de obra femenina se agrupa mayoritariamente en la categoría de artesanos y operarios de hilanderías, confección de vestuario y de calzado. También trabajan en la fabricación de productos alimenticios. Le siguen en importancia en la industria manofacturera las mujeres que se desempeñan como empleadas de oficina.

Es importante señalar que las mujeres profesionales que se desempeñan en este sector representan alrededor de un $4 \%$.

En el rubro de servicios financieros, la gran mayoría de las mujeres, aproximadamente el $70 \%$, ocupan cargos administrativos, le siguen las que realizan tareas menores y las profesionales y técnicas, que en el sector alcanzan a un $14 \%$ aproximadamente.

Las mujeres tienen en promedio una jornada semanal más corta y una tasa de ausentismo mayor que los hombres, observándose en 1994, un promedio de 44.6 horas semanales de trabajo en mujeres y de 47.8 para los hombres. En igual período la tasa de ausentismo fue de 4.3 para las mujeres y de 2.5 para los hombres ${ }^{4}$.

\section{CARACTERÍSTICAS DEL TRABAJO FEMENINO}

En Chile, el proceso de industrialización y la migración desde zonas rurales a las grandes ciudades del país, desencadena que la mujer comience a incorporarse a la vida pública en los años veinte, observándose en ese entonces una tasa de participación femenina de un $20 \%$, la que se mantuvo, con pequeñas variaciones, hasta la década del cuarenta ${ }^{9}$. Fue en ese período donde la mujer comienza a acceder en forma importante a la educación media y en la década del setenta a la educación universitaria; lo que comenzó a aminorar las diferencias educacionales entre los sexos y a incrementar la probabilidad de acceder a nuevas fuentes laborales de mayor status.

El 3 de enero de 1991, es publicada en el Diario
Oficial la ley $\mathrm{N}^{\mathrm{o}} 19.023$, que otorga vida legal al Servicio Nacional de la Mujer. Se crea así, un organismo encargado de velar por la igualdad de derecho y oportunidades para la mujer chilena colaborando con el gobierno en la elaboración, implementación y coordinación de políticas intersectoriales que posibiliten la plena incorporación de la mujer al proceso de desarrollo político, social, económico y cultural del país.

La mujer en el momento actual, accede cada vez más creciente al ámbito público, sin embargo, no están dadas las condiciones sociales, económicas, jurídicas y culturales para que la incorporación sea plena y en igualdad de oportunidades, derechos y deberes.

La sociedad chilena con un modelo de dominio patriarcal, donde culturalmente hay aún diferenciación de roles de acuerdo al sexo de los ciudadanos, las mujeres que tienen un trabajo remunerado presentan mayores dificultades para dedicarse a su trabajo donde juega un rol importante el proceso de socialización. Los resultados de una investigación sobre Mujer y Trabajo ${ }^{10}$, destaca que en la sociedad chilena, el trabajo es el centro de la vida de los hombres, aquello a lo cual dedican el máximo de energía, lo que explica por una parte sus logros profesionales, laborales y su reducida participación familiar.

Las mujeres ${ }^{10}$, en términos de identidad, se definen aún en función del rol tradicional, se dedican menos al trabajo que los hombres, y al perfeccionamiento profesional y tienen logros menores en el ámbito público. Los hombres son educados desde pequeños como seres independientes, cuya misión será producir metas y mantener a la familia. Se estimula en ellos la ambición profesional, el fijarse metas realistas y el desarrollo de virtudes necesarias al logro: perseverancia, sentido de esfuerzo, lucha, responsabilidad.

Las mujeres ${ }^{10}$ son educadas en la ambigüedad, para ser individuos dependientes de sus cónyuges e independientes para enfrentar momentos difíciles. No se favorece su ambición, o se la favorece mientras sea compatible con su rol futuro de madre y esposa. No se les inculca los valores indispensables al logro profesional. Todo ello favorece la crisis vocacional. En las mujeres profesionales se perciben fuertes sentimientos de culpa respecto de sus hijos por el hecho de trabajar. Las mujeres obreras en cambio se sienten orgullosas de contribuir mediante su trabajo al bienestar de sus hijos.

En los hombres ${ }^{10}$ la motivación fundamental para trabajar es de órden económico lo que también se dá en la mujer obrera. En las profesionales priman las motivaciones extra-económicas, ligadas al desarrollo personal y se silencian los asuntos vinculados al dinero. La visión de porvenir de los hombres profesionales y obreros, gira en torno al trabajo. En las mujeres hay dos polos de interés, la familia y el trabajo en un equilibrio 
inestable y a menudo doloroso.

Con la participación d ela mujer en el ámbito productivo; el sistema de roles se encuentra en un proceso de pugna; entre un sistema de roles segregados y roles compartidos.

En el presente ${ }^{9}$, existe dificultad para aceptar los roles compartidos en todos los estratos sociales, sin embargo, la clase media parece ser la que mejor ha asimilado la idea; no así el estrato bajo, donde los roles hombre-mujer suelen ser más rígidos. En este último estrato, la mujer suele incorporarse a una fuente productiva como una estrategia de supervivencia familiar.

Las mujeres trabajadoras en Chile, enfrentan en mayor medida que los hombres algunos obstáculos sea para acceder a un trabajo como en el desempeño del mismo. Entre los más relevantes se pueden destacar:

- Dificultad de acceso al mercado de trabajo, ya que, existen ocupaciones donde hay una marcada preferencia por ocupar mano de obra masculina. Al mismo tiempo las tareas domésticas y el cuidado de los hijos asignados culturalmente a la mujer, dificultan su incorporación. Ya se ha puesto en evidencia que la participación de la mujer con hijos ${ }^{3}$ es significativamente inferior, menos de la mitad que aquellas mujeres sin hijos. Entre las mujeres que tienen mayor educación la tasa de participación sube, pero siempre se da una diferencia importante entre las mujeres con hijos y las que no los tienen.

- Las responsabilidades domésticas no sólo dificultan el acceso de la mujer al trabajo, sino también interfieren con el desempeño de sus actividades laborales, dentro de estas dificultades la más relevante es la que se refiere al cuidado de los hijos. En nuestra cultura todavía se asigna casi exclusivamente a la mujer el cuidado de los hijos, de tal manera que aquellas que se incorporan al mercado laboral, se ven enfrentadas a tener que compatibilizar esa responsabilidad con el desempeño de su rol de trabajadora, lo que no siempre resulta compatible o al menos es muy dificil. Esto se agrava dada la concepción predominante en nuestro país que es un problema que debe ser resuelto en forma privada. De hecho la actual legislación contenida en el código del trabajo ${ }^{1}$ sólo da respuestas parciales al problema del cuidado infantil, estableciendo que tendrán derecho a sala cuna para sus hijos menores de dos años las trabajadoras de cualquier edad o estado civil que laboran en establecimiento con veinte o más mujeres. Tal normativa deja sin solución la mayor parte del problema, al no considerar el cuidado de los hijos mayores de dos años, de las trabajadoras que laboran en instituciones con menos de veinte mujeres y de aquellas trabajadoras que se desempeñan en el sector informal de la economía o en tareas de temporárias. Por tanto, una medida legal para favorecer a la trabajadora, se convierte en causa de discriminación en el mercado laboral, de tal manera el tema del cuidado infantil afecta directamente el empleo femenino y no así al masculino.

- Estudios demuestran que por trabajos de igual valor, es posible observar que las mujeres en forma general perciben un salario inferior al de los hombres; situación que afecta a el $69 \%$ de las mujeres cuyos ingresos corresponden a salarios; sólo el $31 \%$ de los ingresos femeninos corresponden al trabajo independiente. El salario de los hombres son el $51 \%$ de sus ingresos y el $49 \%$ proviene de su trabajo independiente ${ }^{4}$. Esta discriminación se acentúa a medida que los niveles educacionales son mayores, de tal manera que la brecha salarial es más alta en los niveles superiores de la educación. Así las profesionales y técnicas, según encuesta Nacional de Empleos octubre- diciembre $1992^{9}$, percibían en promedio un $45 \%$ de las remuneraciones que perciben los hombres, mientras que las trabajadoras de servicios personales y afines recibían un $64.6 \%$ del salario que recibían los varones en esa misma categoría. El informe del Servicio Nacional de la Mujer del año $1995^{8}$, relativo al ingreso de las mujeres en Chile, señala que el ingreso de las mujeres es inferior al de los hombres en casi todas las ramas de actividad económica y en dos de ellas -industria y finanzas, apenas superan el $50 \%$ del ingreso masculino; ramas en que más creció el empleo femenino. El ingreso medio mensual de los asalariados aumentan con la edad hasta el tramo entre 35 y 54 años para las mujeres y 45 a 54 años para los hombres. El máximo ingreso alcanzado por las mujeres entre los $35 \mathrm{y}$ 44 años es comparable al que alcanzan los hombres entre los 25 y 29 años.

En el sector público en el año $1994^{4}$, predominan los hombres en los grados más altos en la escala de remuneraciones. Como resultado de esta distribución, las mujeres ganan, en promedio, un $23 \%$ menos que los hombres y la mayor diferencia se produce en la categoría de profesionales.

La discriminación aumenta con la edad ${ }^{4}$. Las menores de 35 años ganan, en promedio, 22\% menos; las de 36 a 55 años 30\% menos; las de 56 a 60 años $28 \%$ menos y las mayores de 60 años $38 \%$ menos. La experiencia asociada a la edad y la mayor preparación asociada al título profesional o técnico, no conducen a una situación más igualitaria en términos de remuneraciones sino, por el contrario, a una mayor discriminación.

- Los cargos de dirección en las diferentes ramas de la actividad económica son en su mayoría ocupados por hombres. Para la mujer es dificil acceder a estos cargos por diferentes razones derivadas en su mayoría del modelo cultural predominante en nuestra sociedad. Un estudio realizado en $1992^{4}$ en 490 empresas de gran tamaño en Chile, fue posible observar que en 285 de ellas, 
(58\%), había al menos una mujer en cargos ejecutivos, en las restantes 205 empresas, no había ninguna. En el primer nivel jerárquico de las empresas, director superior, gerencia general había un 6\% de mujeres; en el segundo nivel correspondiente a gerencias se constató un $10 \%$; en el tercer nivel jerárquico subgerencias y jefatura superior un $19 \%$ de mujeres; en el cuarto nivel jerárquico jefaturas y otros un $23 \%$ y en un quinto nivel de mandos medios, nivel operativo un $26 \%$ de mujeres.

- La participación sindical de las mujeres es incipiente, siendo un tercio de los asalariados; constituyen ellas sólo un séptimo de los dirigentes sindicales. En 1993, a nivel de todo el país con un total de 19.479 dirigentes sindicales sólo el $15 \%$ eran mujeres, asumiendo como presidenta un $12 \%$; como secretaria $17 \%$; en tesorería un $16 \%$ y como directora un $9 \%$.

Las razones de estas desigualdades podrían tener explicación en los valores y creencias tradicionales arraigadas en la sociedad, donde atribuyen a los sexos funciones diferenciadas acentuando las actividades socializadoras, afectivas y protectoras como propias de la mujer y aquellas que se vinculan al poder, ingresos monetarios, prestigio social, liderazgo institucional como propias del hombre.

\section{COMENTARIO}

El presente estudio posibilitó conocer la situación laboral de la mujer en Chile; su compleja problemática al incorporarse en el mundo público. La fuerza laboral en Chile ha ido creciendo progresivamente desde una tasa de 24.1 en 1970 a 41 en 1994, en edades entre 20 y 64 años.

La participación de la mujer en el mundo del trabajo sea formal o informal, no se ha visto vinculada a la creación de mecanismos sociales, legales y de salud que permitan a la mujer compatibilizar sus roles socialmente asignados del ámbito privado como madre, esposa y dueña de casa con el rol productivo que ha ido asumiendo.

La inserción laboral femenina se ha producido en ocupaciones que se consideran una extensión de los roles reproductivos, concentrándose alrededor del 50\% en el área de servicios percibiéndose una desvalorización de esas ocupaciones por parte de la sociedad.

Requiere de análisis y estudio la situación manifiesta de descriminación salarial entre los sexos, encontrándose diferencias en todas las categorías ocupacionales en donde participa la mujer, siendo más marcada en aquellas donde se requieren niveles superiores de educación.

Para una plena participación de la mujer se hace indispensable acciones intersectoriales que permitan la igualdad de oportunidades y de reconocimiento del trabajo de la mujer entre las más relevantes se proponen: modificaciones legales y en la práctica jurídica para resguardar la igualdad de derecho entre mujeres y hombres; favorecer la participación equitativa de mujeres y hombres en los procesos educativos y de producción y transmisión del conocimiento; promover una imagen social plural y no discriminatoria de las mujeres en la cultura y la comunicación; facilitar el acceso y mejorar la posición de la mujer en el mercado de trabajo; mejorar la situación de salud de la mujer; facilitar la igualdad de oportunidades a todos los tipos de familias e impulsar a que ellas constituyan instancias de generación de igualdad de derechos y oprtunidades para hombres y mujeres; para poder construir una sociedad con mayores niveles de equidad social.

\section{WOMAN LABOUR FORCE IN CHILE: DATA AND CHARACTERISTICS}

The present article shows us some sociodemographic characteristics of the women labour force in Chile, and the difficulties they have to accede and to maintain their labour source due to gender conditions.

KEY WORDS: woman, labour force, Chile

\section{REFERENCIAS BIBLIOGRÁFICAS}

01. CHILE. Encuesta nacional de empleos. Julio Septiembre, 1993.

02. CHILE. Encuesta nacional de empleos. Septiembre - Noviembre, 1993.

03. CHILE. Instituto Nacional de Estadísticas. Censo poblacional, 1992.

04. CHILE. Instituto Nacional de Estadística. Mujeres y hombres en Chile: cifras y realidades. Santiago, 1995.
05. CHILE. Servicio Nacional de la Mujer. Salud de la mujer en Chile. Santiago, 1992. documento n.22.

06. DIAZ, X. et al. Mujer, trabajo y familia: el trabajo a domicilio en Chile. Santiago: CEM, 1996.

07. HENRIQUEZ, H.; PEREZ, E. La subestimación de la participación femenina en las actividades económicas: encuesta suplementaria en mujeres inactivas. Santiago: SERNAM, 1994.

08. ILLANES, M. Algunas características del mercado de trabajo y la participación de la mujer. Santiago: SERNAM, 1995. 
09. NACIONES UNIDAS. Comisión Económica para América Latina y el Caribe. Cambios en el perfil de la familia. Santiago, 1993.
10. ROSSETTI, J. Emancipación y trabajo un camino por hacer. Santiago: Centro de Investigación y Desarrollo de la Educación, 1984. 